Brit. J. industr. Med., 1959, 16, 311.

\title{
LATE CONSEQUENCES OF THE NEUROLOGICAL FORMS OF DECOMPRESSION SICKNESS
}

\author{
BY \\ I. RÓZSAHEGYI \\ From the Institute of Industrial Medicine, Budapest, Hungary
}

(RECEIVED FOR PUBLICATION OCTOBER, 1958)

\begin{abstract}
Various neurological forms of decompression sickness are classified and an inquiry two and a half to five and a half years after the acute illness is reported.

One hundred subjects with decompression sickness of the central nervous system were examined.

In more than half of the cases the clinical picture was that of chronic encephalomyelopathy, vegetative neurosis, or psychosomatic symptoms. Symptoms were found in three-quarters of the cases, and objective signs in the same proportion. Fourteen patients were unable to work two and a half to five and a half years after the acute illness, and only 13 of 100 patients were reported to be reasonably well.

The best and only adequate treatment for decompression sickness is recompression. After recompression the prognosis for recovery is increasingly poor the longer time passes, and after the first six weeks there is no hope of a perfect recovery.
\end{abstract}

The pathological changes in the spinal cord caused by decompression trauma in divers and caisson workers have already been described, as also have cases of damage to the brain, but the characteristics of the neurological form of decompression sickness are not as yet generally known. The future of these patients is also uncertain because histories of individual cases are not sufficient to make any general prognosis.

During the building of the metro in Budapest, all cases of decompression sickness in which the patients were unable to work for three days or more were referred to us. It was therefore possible for us to make a systematic study of caisson sickness and to analyse the results of our investigation (Rózsahegyi and Soós, 1956), and in a period of from two and a half to five and a half years after the acute illness, I have followed up 100 patients to see if $I$ could evaluate the prognosis of decompression sickness affecting the central nervous system.

Each manifestation of decompression sickness of the central nervous system is in effect a multifocal lesion, but it is possible to differentiate between four types, namely, (1) the syndrome of multiple focal injuries of the whole of the central nervous system; (2) the syndrome in which there are multiple lesions in the cerebrum and the upper brain-stem; (3) the syndrome of injury to the medulla, pons, and cere- bellum; and (4) the syndrome exhibiting spinal lesions. The essential features of these various forms will be described briefly here; for greater detail our monograph should be consulted (Rózsahegyi and Soós, 1956).

\section{Neurological Forms}

(1) Multiple Focal Injuries of the Whole Central Nervous System.-The illness begins acutely with a general collapse, central hypoxaemia, or with signs of "pseudo-Ménière's disease", by which we mean dizziness caused by a lesion of the bulbar vestibular nuclei, that is, of central origin as opposed to the Ménière syndrome caused by a lesion of the labyrinth of peripheral origin. The onset is swift. In slight cases, when there is no loss of consciousness, the signs of paraplegia and autonomic disturbances are unequivocal (sweating, dizziness, pallor). Complete recovery after recompression is rare, and in most cases residual signs in the form of a secondary disseminated encephalomyelopathy can be observed. In one-third of the cases a vegetative neurosis and in another third pathological, hysterical changes of the personality are seen as late complications.

(2) Multiple Lesions in Cerebrum and Upper Brainstem.- Regional cortical aeropathy is a not very frequent event in caisson workers but that of the 
upper brain-stem, diencephalon, and pontomesencephalon is often found. It generally begins without premonitory symptoms with sudden collapse and unconsciousness; if there are warning symptoms, these consist of blurring of vision (retinal aeropathy), but not systemic dizziness, headache, and serious vegetative symptoms. Focal symptoms remain. A psychosomatic syndrome is in more than half of these cases a frequent late sequel. The acute illness is often followed by a vegetative neurosis.

(3) Injury to the Medulla Oblongata, Pons, and Cerebellum.-There are cases in which an isolated lesion of the bulbar vestibular nuclei may be observed. In those cases, the after-effects are noted as an instability of the vestibular mechanism. In other cases of this group there are, mostly in addition to the vestibular defect, signs of damage to cranial nerve nuclei and the long nerve tracts. Both kinds of lesion often appear in the form of central collapse with dizziness, which cannot be differentiated in the acute phase from the Ménière syndrome of peripheral origin. We have described this as the pseudo-Ménière syndrome. Later it is possible to differentiate between the two forms. Cases of "peripheral" Ménière syndrome recover without lasting damage or else the typical symptoms of the chronic permanent Ménière syndrome persist, mostly with serious damage to the cochlear apparatus. Cases of the pseudo-Ménière syndrome are left with dizziness but without any damage to hearing. The otoneurological state is normal in these cases or there is evidence of a central lesion. A frequent consequence is a vegetative neurosis with a tendency to fainting.

A lesion in the cerebellum is not frequent but is serious.

Cases of massive air embolism of the vital bulbar centres may end fatally.

(4) Spinal Lesions.-It is necessary to distinguish those cases with a lesion below the tenth thoracic segment. After a prodromal paraesthesia an acute atonic paraplegia follows, and, in spite of recompression, there is retention of urine for several days. A very frequent consequence is complete or partial impotence. The paralysis of the lower limbs is followed by slight spasticity after a shorter or longer period. Corresponding to the position of the lesion there are residual sensory disturbances.

Tetraplegia is characteristic of multifocal lesions of the spinal cord, and develops with frightening speed although the patient remains conscious. The result is disseminated myelopathy, or, if the brain is also affected, encephalomyelopathy. Also there are cases of primary chronic myelopathy or encephalomyelopathy in which the development of decompres- sion trauma is insidious. No acute illness after decompression injury has been noted. The diagnosis is made in these cases during a routine examination or for another illness and is based on focal symptoms.

In addition to primary and secondary myelopathy and encephalomyelopathy there are, as late consequences of the neurological appearance of decompression sickness, certain clinical signs, namely, a vegetative neurosis and a pathological change of personality or pseudo-neurasthenia or hysteria.

The vegetative neurosis is mostly associated with focal symptoms of the bulb and pons. It is seen after the acute damage to the upper brain-stem, cerebral, or multifocal cerebrospinal lesions, but never after an isolated lesion of the spinal cord. For this reason we are of the opinion that it is not of psychological origin but is a consequence of an organic lesion of the autonomic centres around the third and fourth cerebral ventricle and the aqueduct of the mid-brain. The most frequent complaints are anorexia, headache, disorders of sleep, lassitude. The most frequent symptoms are the signs of derangement of the whole of the autonomic nervous system (tremor, dermographismus, exaggerated reflexes, dilated pupils, often a slight exophthalmos).

The psychosomatic syndrome can be seen in about a third of the cases of neurological decompression sickness. These cases all have a lesion of the brainstem ganglia and of the diencephalon, and the syndrome never follows an isolated lesion of the spinal cord. The changes in personality are of particular importance. Men who were quiet before injury become extremely irritable and uncontrolled. Pathological drunkenness and alcohol intolerance are also frequent.

The neurological enquiry was made after the acute decompression sickness as follows:-

$$
\begin{aligned}
& \text { In } 3 \text { cases after } 2 \frac{1}{2} \text { years } \\
& \text { In } 3 \text { cases after } 3 \text { years } \\
& \text { In } 3 \text { cases after } 3 \frac{1}{2} \text { years } \\
& \text { In } 18 \text { cases after } 4 \text { years } \\
& \text { In } 34 \text { cases after } 4 \frac{1}{2} \text { years } \\
& \text { In } 36 \text { cases after } 5 \text { years } \\
& \text { In } 3 \text { cases after } 5 \frac{1}{2} \text { years }
\end{aligned}
$$

The ages at the time of the investigation were as follows:-

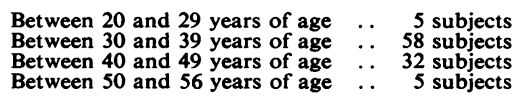

The clinical picture in the different appearances of the acute illness is shown in Table 1. No definite defect remained in about one half of the cases $(41 \%)$; the fewest cases without any residual defects were those of the multifocal cerebrospinal lesions $(24.39 \%)$. This too was the only group of patients left with two (two cases) or three (one case) definite symptoms. 
TABLE 1

RELATION OF CHRONIC CLINICAL PICTURES TO FORM OF ACUTE ILLNESS

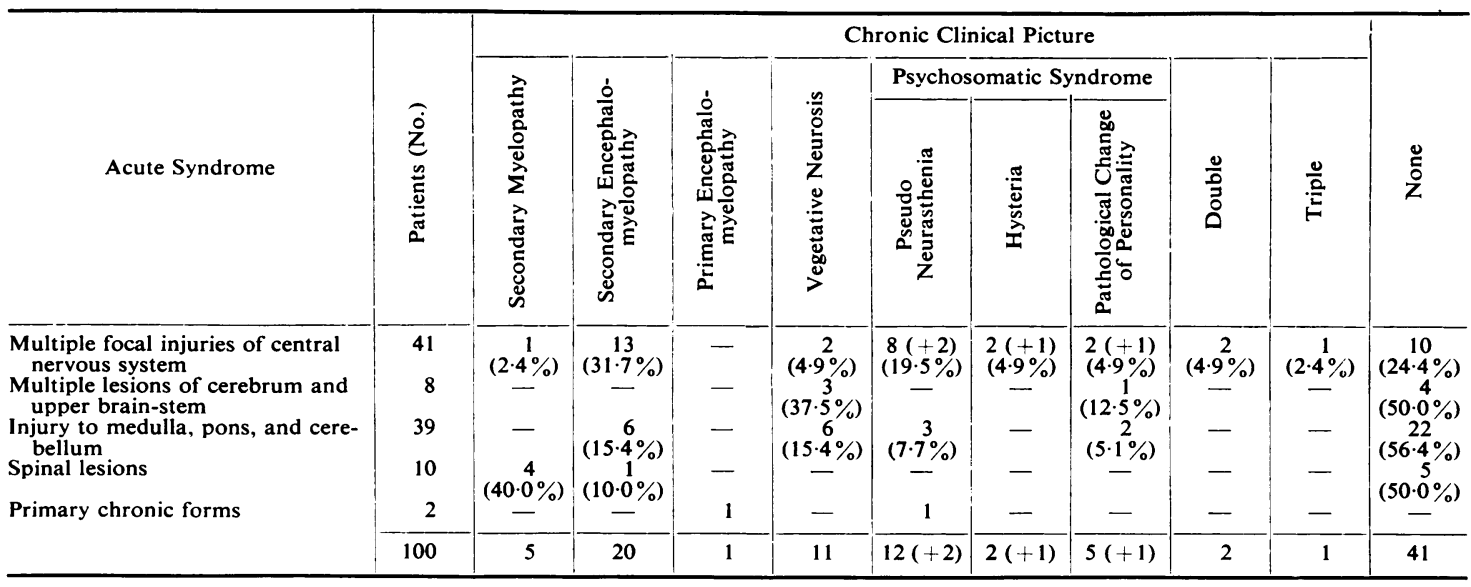

TABLE 2

SYMPTOMS

\begin{tabular}{|c|c|c|c|c|c|c|c|c|c|c|c|c|}
\hline Acute Syndrome & 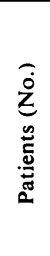 & 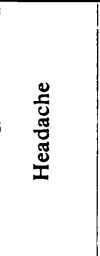 & 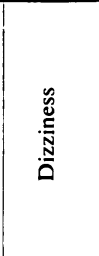 & 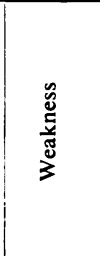 & 总 & 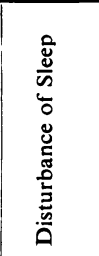 & 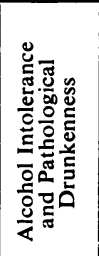 & 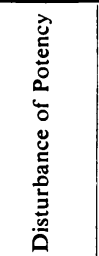 & 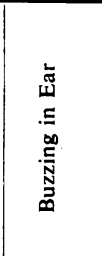 & 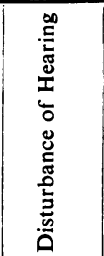 & 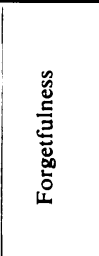 & 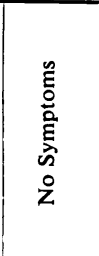 \\
\hline $\begin{array}{l}\text { Multiple focal injuries of } \\
\text { central nervous system } \\
\text { Multiple lesions of cere- } \\
\text { brum and upper brain- } \\
\text { stem }\end{array}$ & $\begin{array}{r}41 \\
8\end{array}$ & $\begin{array}{c}19 \\
(46 \cdot 2 \%) \\
3 \\
(37.5 \%)\end{array}$ & $\begin{array}{c}25 \\
(60 \cdot 8 \%) \\
2 \% \\
(25.0 \%)\end{array}$ & $\begin{array}{c}8 \\
(19.5 \%) \\
1 \\
(12.5 \%)\end{array}$ & $\begin{array}{c}21 \\
(51 \cdot 1 \%) \\
2 \% \\
(25 \cdot 0 \%)\end{array}$ & $\begin{array}{c}19 \\
(46 \cdot 2 \%) \\
-\end{array}$ & $\begin{array}{c}6 \\
(14.6 \%) \\
2 \% \\
(25.0 \%)\end{array}$ & $\begin{array}{c}16 \\
(38.9 \%) \\
-\end{array}$ & $\begin{array}{c}5 \\
(12.2 \%)\end{array}$ & $\begin{array}{c}8 \\
(19.5 \%) \\
-\end{array}$ & $\begin{array}{c}7 \\
(17.0 \%)\end{array}$ & $\begin{array}{c}7 \\
(17.0 \%) \\
4 \\
(50.0 \%)\end{array}$ \\
\hline $\begin{array}{l}\text { Injury to medulla. pons, } \\
\text { and cerebellum } \\
\text { Spinal lesions } \\
\text { Primary chronic forms }\end{array}$ & $\begin{array}{r}39 \\
10 \\
2\end{array}$ & $\begin{array}{c}20 \\
(51 \cdot 3 \%) \\
3 \% \\
(30 \cdot 0 \%) \\
1\end{array}$ & $\begin{array}{c}19 \\
(48 \cdot 7 \%) \\
2 \% \\
(20 \cdot 0 \%) \\
1\end{array}$ & 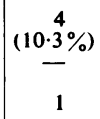 & $\begin{array}{c}15 \\
(38 \cdot 5 \%) \\
2 \% \\
(20 \cdot 0 \%) \\
-\end{array}$ & $\begin{array}{c}10 \\
(24 \cdot 3 \%) \\
2 \% \\
(20 \cdot 0 \%) \\
-\end{array}$ & $\begin{array}{c}7 \\
(18.0 \%) \\
1 \% \\
(10.0 \%) \\
-\end{array}$ & $\begin{array}{c}8 \\
(20.5 \%) \\
3 \% \\
(30.0 \%) \\
1\end{array}$ & $\begin{array}{c}3 \\
(7 \cdot 3 \%) \\
-\end{array}$ & $\begin{array}{c}3 \\
(7 \cdot 3 \%) \\
-\end{array}$ & $\begin{array}{c}3 \\
(7 \cdot 3 \%) \\
1 \% \\
(10.0 \%) \\
-\end{array}$ & $\begin{array}{c}9 \\
(20.4 \%) \\
6 \\
(60.0 \%) \\
-\end{array}$ \\
\hline Primary chronic forms & 100 & 46 & 49 & 14 & 40 & 31 & 16 & 28 & 8 & 11 & 11 & 26 \\
\hline
\end{tabular}

A vegetative neurosis was the most frequent final consequence after the acute illness of a lesion of the upper brain-stem: three-quarters of these cases were left with sequelae.

The different forms of the psychosomatic syndrome persisted after several years only in such cases in which the upper segments of the central nervous system were affected, but never after the lesion of the spinal cord. This syndrome was also most common in the first of our groups, that exhibiting multiple focal lesions of the whole of the central nervous system.

For a long time after lesions of the spinal cord neither a vegetative neurosis nor a psychosomatic syndrome could be observed.

Table 2 summarizes symptoms. There were no symptoms several years after the illness in more than half of the cases of the fourth group (spinal lesions), in half of the second group (lesions of the cerebrum and upper brain-stem), but in the first and third groups only one-fifth of the cases were without symptoms. The most frequent were dizziness $(49 \%)$, headache $(46 \%)$, and increased irritability $(40 \%)$.

The dizziness was continuous only in a few cases, but in most it was brought on by change of position or it appeared in paroxysms. Many patients reported dizziness while travelling. Headaches were seldom continuous; most patients complained about frequently returning headache. Sleep was often disturbed: most patients complained of insomnia, but in other cases of sleepiness, and in a few cases of both disturbances with inverse sleep rhythm.

Sexual potency was disturbed in 28 cases. In seven of the 16 cases of the first group the impotence 
TABLE 3

SIGNS

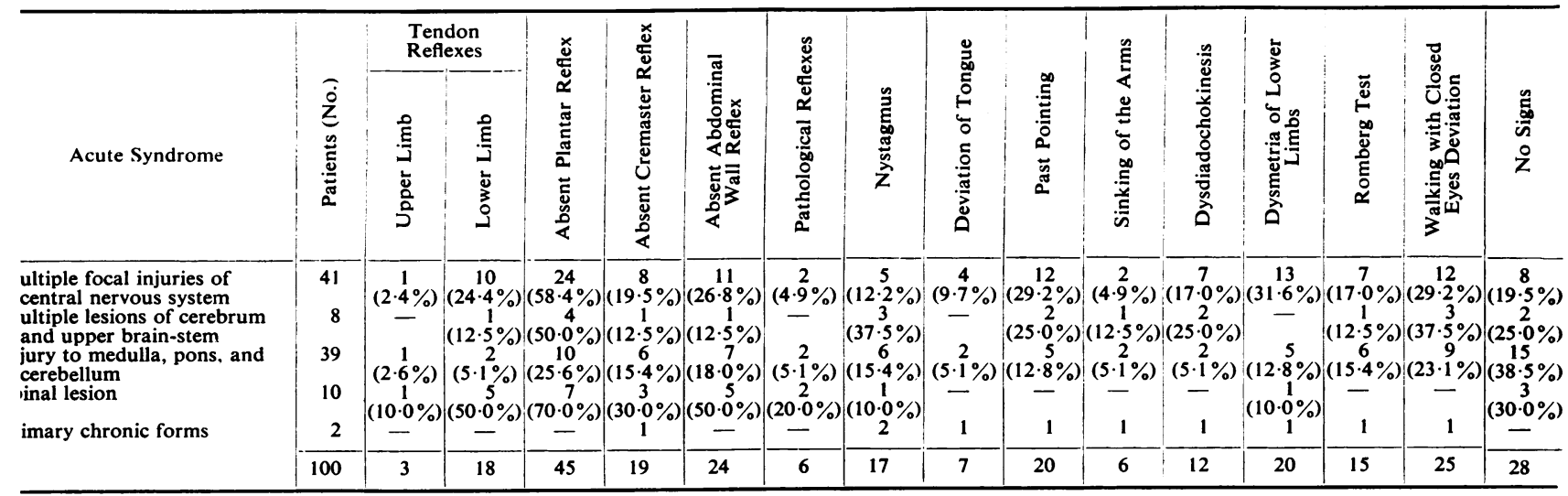

was complete, in nine cases partial (with unsatisfactory erection or diminished sexual desire). The eight cases of the third group were all partial, the three cases of the fourth group all complete.

Other complaints were only sporadic.

Among the objective findings (Table 3) the most frequent was the absence of the plantar reflex, not so frequent the absence of the abdominal reflex. Unsteadiness in walking with closed eyes was observed in every fourth case, faulty postural sense of the lower extremities and past pointing in every fifth case. The tendon reflexes showed abnormalities in the lower extremities in 18, and in three cases in the upper extremities being increased, absent, or different on the two sides. Pathological reflexes were seen only in six cases examined a long time after the acute phase. Other neurological signs were sporadic.

Most symptoms and most objective findings were in the first group, namely, in the syndrome of multifocal aeropathy of the whole of the central nervous system as was expected. Even the fact of multiple foci does not make it possible to relate the single complaints and symptoms to particular structures. This is possible only in the more differentiated forms. Headache, dizziness, increased irritability, and disturbances of hearing and of equilibrium seem to be related to lesions of the cerebellum, pons, and the bulb; weakness, alcohol intolerance, nystagmus, and disturbances of motion to those of the cerebrum and upper brain-stem. Disturbances of potency, absence of skin reflexes (plantar, cremaster, abdominal) and difference or absence of tendon reflexes and pathological reflexes (Babinsky, Oppenheim) are related to lesions of the spinal cord.

The course of the neurological form of decompression sickness is not invariable. There are cases in which the recompression in the medical lock was fully effective and they showed no defect immediately after the decompression or later.

M.I. had decompression sickness at the age of 26 . After decompression he experienced irritation and numbness in the buttocks and thighs. He was at once given recompression in the medical lock at a pressure of 2.9 atmospheres and symptoms ceased, and four and a half years later he has no signs or symptoms.

V.K. had caisson sickness at the age of 26 . He had been working at a pressure of 1.5 atmospheres. He felt dizzy and his feet were numb in the caisson during decompression. During the recompression, which was carried out immediately after a hot shower and at a pressure of 2.9 atmospheres, the symptoms disappeared, and after eight hours he left the medical lock without any symptoms. Four years later he has no signs or symptoms.

In other cases the symptoms disappeared in the medical lock during the recompression and they did not reappear immediately afterwards, but later either the same or new symptoms were experienced and with them corresponding objective signs.

M.G., aged 39, on July 14, 1955, had decompression sickness. He had been working for three and a quarter hours at a pressure of 1.4 atmospheres. During the decompression the right arm began to twitch and later the right arm and lower limb became numb and insensible and painful. The pressure in the medical lock was immediately raised again but he became dizzy and pale and his face was paralysed; he was unable to speak and lost consciousness. In the medical lock he regained consciousness and all symptoms disappeared at a pressure of $2 \cdot 2$ atmospheres. Seven hours later he left the medical lock without symptoms. The next day he had headache, but no other symptoms and the neurological examination yielded no pathological signs. At examination on August 29 he also had no complaints and no neurological symptoms. On November 1 he complained of headache and dizziness, but had no objective symptoms except 
anaesthesia of the vestibular nerve and a hypoaesthesia of the cochlear nerve. He complained further about headache, weakness, and uncertainty in walking, without other objective findings. Not before December 14, 1955, however, did he show neurological symptoms. Then he had a dysdiadochokinesia of the left arm and absent abdominal reflexes. On March 22, 1956, he reported increased irritability, impatience, and impotence. Neurological and otoneurological findings were unchanged till the examination in February, 1958. Then he reported numbness and dull pains in the feet and from time to time he had fever. He still had dysdiadochokinesia of the left arm, absent abdominal reflexes, and an absent right patellar reflex.

In the following case, over a period of several months, chronic encephalomyelopathy and a pathological change of personality developed, and seems to be fixed.

St. F., aged 46, on December 30, 1955, had worked for six hours at a pressure of 1.6 atmospheres and three hours after ceasing work he had decompression sickness. First he had a feeling of numbness in the sacral region and in the thighs, later he became anaesthetic from the hips downwards. By recompression up to 2.8 atmospheres he lost these symptoms and left the medical lock seven hours later apparently well. The next day he again felt numbness in the feet and reported the same symptoms at examination on January 3,1956 . The objective findings were a very marked diminution of reflexes of the lower limbs, hypoaesthesia on the right side from $D_{6}$, on the left from $D_{10}$ downwards, absent cremasteric reflexes and absent right plantar reflex. There was a pronounced ataxia of the right lower limb. The next day he had disturbances of micturition, so that he had to be admitted to hospital. He was discharged improved after 24 days. On January 26 the right Achilles jerk was absent and also the right plantar reflex and there was ataxia and adynamia of the right lower limb. In the middle of February the patellar and tendon of Achilles reflexes disappeared. On March 6 he reported improvement, and his only complaint was a stabbing pain in the spine. Only the plantar reflex was positive. At the end of March he was without complaints with unchanged objective findings.

Two years later, on February 11, 1958, he complained of weakness of the feet, discomfort in rassing water, temporary incontinence, and complete impotence. He had dysmetria of the lower limbs (right worse than the left), absence of the patellar and tendon of Achilles reflexes and of the cremasteric reflexes and diminished plantar reflexes.

This is a case of the slow evolution of damage to the spinal cord, which leaves behind a definite defect with impotence, temporary incontinence, and weakness of the lower limbs.

In some cases, perhaps due to faults in the technique of recompression, the symptoms did not disappear in the medical lock but persisted or got worse. In the acute phase it is remarkable that the subjective complaints and the objective signs show considerable variation. They diminish or disappear for hours or even days then return, perhaps aggravated. This variability in the severity of signs and symptoms may continue for several weeks or months. Convalescence in the slight cases lasts some days, in the majority of the cases three to six months. After a year there is generally no hope of recovery, but in some exceptional cases recovery is possible even after several years.

M.A., aged 22 years, on November 10, 1953, had worked for three and a half hours under pressure and then, after a break of $\mathbf{2 0}$ minutes, for a further four hours. About $\mathbf{4 5}$ minutes after the second working period he developed a headache and in about an hour felt ill, lost consciousness, and needed recompression. After seven and a half hours' treatment he felt weak and giddy though the pressure had been raised to $2 \cdot 8$ atmospheres. He had a multifocal air embolism of the central nervous system with a lesion of the cerebellum. After recompression he still had paroxysms of dizziness and could not travel without becoming giddy. He was impotent. He showed a positive Romberg test, ataxia of the trunk, nystagmus to the left, past pointing, ataxia of the inferior extremities, and exaggerated tendon reflexes. His symptoms got worse, together with disturbances of the autonomic nervous system and he became weak.

In November, 1954, the dizziness was still constant but less severe. At this time there was deviation of the tongue to the right, dysdiadochokinesia of the right arm, and the nystagmus was now observed in both directions; paresis of the right abducens was found also. Walking with closed eyes could not even be attempted because the patient fell at once.

In November, 1955, there was some change in his symptoms. The paroxysms were less frequent, but he was feeling a continuous sensation of a pull to the right. He slept 14 to 16 hours a day. The ataxia of the trunk was not now noted, but nystagmus, dysdiadochokinesia, past pointing, and a positive Romberg test persisted. The patellar reflex was on both sides exaggerated. He was unfit for work.

In August, 1956, he had sufficiently improved to be able to perform an administrative job, and in October, 1956, he dared to begin to study law. He was still prone to dizziness, but not so much as before; with closed eyes he felt unsafe. Any slight physical effort also provoked weakness.

In May, 1958, he had only the sensation of a slight pull to the right; he did not have paroxysms of dizziness any longer, and felt unsafe only during travel. The sleepiness and the impotence stopped. He felt well and his only complaint was that he could not ride a cycle. The tongue still showed deviation to the right, there was a nystagmus in both directions (to the left slight, to the right marked), and past pointing with both hands, the right more pronounced than the left. Dysdiadochokinesia was not present. The Romberg test was positive, but not so pronounced as before. Walking with closed eyes was now possible, but with a strong deviation to the right. 
The tendon and skin reflexes were normal except the plantar reflex, which was absent. There was poor postural sense of the right lower limb, on the left there was no abnormality.

In this case three years were necessary to obtain a significant improvement and only four years after the acute illness could the patient be regarded as well, though with residual defects.

E.K., aged 38, on January 30, 1954, had a multifocal aeropathy of the central nervous system. He had worked for six hours at a pressure of 1.6 atmospheres and one hour after stopping work he had pain in the right arm and leg. Later he developed paraesthesiae, formication, and a feeling of weakness. He was at once recompressed and raised to a pressure of 2.7 atmospheres. His symptoms ceased and he left the air chamber after seven hours with only slight pain in the limbs. He had the sensation of palsy and of paraesthesia in the feet and pains in the limbs of the left side. At the acute phase he showed a sinking tendency in the left arm, nystagmus to the left, and deviation to the left on walking with closed eyes. The otoneurological examination showed a serious lesion of both branches of the eighth cranial nerve on both sides. This lesion healed in 12 weeks, but the symptoms did not become less grave and dizziness and nausea were added, particularly, if he travelled or even if he only looked at moving vehicles. He complained of impotence. In June, 1954, the complaints were unchanged but in the left arm there was weakness and a significant dysdiadochokinesia, absence of the patellar reflexes, and ataxia of the right lower limb. The symptoms lasted, with fluctuations, a whole year; there were disturbances of the autonomic nervous system and headache. The objective findings were also unchanged and not before March, 1955, was there a slight improvement. At this time he was able to travel without dizziness and nausea and to try some easy physical work. In July he tried harder work, but he fell repeatedly and lost consciousness; he had paroxysms of dizziness. His potency returned at this time.

At the end of 1956 he began work in a coal-mine. In February, 1957, he had his last paroxysm of dizziness, and at the end of March his potency began once more to decrease. In June, 1957, he had no symptoms, and except for the absence of the right plantar reflex there are no objective findings. Potency returned after injections of yohimbin, strychnine, and tocopherol.

This patient had needed three years to recover from air embolism of the central nervous system though his symptoms at the end of that time were slight.

In the cases which were free from complaints and symptoms two and a half to five and a half years after the acute illness one subject was free immediately, four subjects were free one week later, five two weeks later, two three weeks later, one four weeks later, and one subject was well six weeks later. Those patients who had complaints six weeks after the decompression still have residual symptoms after several years. The outlook for a complete recovery must be evident in the first six weeks.

The tendency to recover after decompression sickness of the central nervous system is good, but in about three-quarters of the cases there remain objective symptoms or signs which prove lasting damage to these tissues. There are only 14 of 100 subjects who show no signs or symptoms more than two years after the acute illness, and if we disregard slight symptoms or signs this number is not even half the cases $(36 \%)$. Of 100 subjects, there were 14 who, up to the time of this investigation, were not able to work. From this point of view, each case of decompression sickness of the central nervous system is to be regarded as grave and the prognosis as dubious.

The first group has the worst prognosis, and there were only three cases $(7.32 \%)$ in which no complaints and no objective symptoms were found: in nearly all the cases a definite lesion of the central nervous system remained. The prognosis of lesions of the spinal cord is better: in nearly one-third of the cases recovery is complete. The prognosis of the other groups is between these two.

How may the prognosis of decompression sickness of the central nervous system be ameliorated? First it is essential to take all preventive measures,

TABLE 4

\begin{tabular}{|c|c|c|c|c|}
\hline \multirow{3}{*}{\multicolumn{2}{|c|}{ At Time of Epicritic Investigation }} & \multicolumn{3}{|c|}{ After Discharge from Medical Lock } \\
\hline & & \multicolumn{3}{|c|}{ Symptoms } \\
\hline & & None & Uncertain & Persistent \\
\hline & $\begin{array}{l}\text { Patients } \\
\text { (No.) }\end{array}$ & 28 & 18 & 52 \\
\hline $\begin{array}{l}\text { No symptoms or signs } \\
\text { Slight symptoms and signs } \\
\text { Severe symptoms and signs }\end{array}$ & $\begin{array}{l}15 \\
69\end{array}$ & $\begin{array}{l}(64.3 \%) \\
(33.3 \%)\end{array} 9^{(17.9 \%)}$ & $\frac{(21.4 \%)}{(26.7 \%)} 4^{3} 4_{(16.7 \%)}^{(26.7 \%)}$ & $\begin{array}{ll}(14.3 \%) & 2^{(3.9 \%)} \\
(40.0 \%) & 6^{(11.5 \%)} \\
(63.8 \%) & 44{ }^{(84.6 \%)}\end{array}$ \\
\hline
\end{tabular}


which consist in preventing fluctuations of pressure in the caissons, keeping the climatic environment even, and ensuring conditions of safe decompression. Medical prevention should ensure the selection of fit subjects for caisson work and the elimination of those who exhibit an increased predisposition to decompression sickness. The subject who repeatedly falls sick with the same type of decompression sickness is particularly prone to the neurological form. Moreover, decompression sickness of the central nervous system leaves the patient with an increased predisposition to it and it is not advisable for him to return to caisson work.

The prevention of definite defects of the central nervous system is the correct therapy of every case of decompression sickness, and the only treatment is recompression, the most important part of which is the decompression. It is wise in serious cases to order a prolonged decompression, the American "overnight soak", even if it lasts for 24 hours or more.

The auspices for recovery after decompression are not good. Of those who were free from complaints after decompression in the medical lock, two and a half to five and a half years after the acute illness, more than half were free from complaints but nearly half of the cases had either serious signs or symptoms. In those cases in which the recompression was ineffective and complaints remained after leaving the medical lock, not one in 25 patients was healthy after some years, and in $84.6 \%$ of the cases there were serious signs or symptoms (Table 4).

All other treatment is of secondary significance. It is usual to give vasodilators, such as nicotinic acid, papaverine, and, to assist the regeneration of nervous elements, vitamin $B$.

Nicolas and his colleagues used intravenous injections of procaine in the therapy of acute osteoarthralgy (bends) with good results. We tried this treatment in neurological cases and gained the impression that, if applied early enough, it is useful. We gave $10 \mathrm{ml}$. of the $1 \frac{1}{2}$ to $2 \%$ solution slowly intravenously. The cases are too few to form a definite opinion.

\section{REFERENCE}

Rózsahegyi, I., and Soós, I. (1956). Caissonkrankheit und Zentralnervensystem [Arbeitsmedizin, Heft 30.] Barth, Leipzig. 